Acta vet. scand. 1967, 8, 287—289.

Brief Communication

\title{
THE LINEAR DISTRIBUTION OF TWO OESOPHAGOSTOMUM SPECIES IN THE INTESTINE OF THE PIG
}

In both Denmark and Scotland the domestic pig, Sus scrofa domestica, is frequently parasitised by two species of the nematode genus Oesophagostomum: Oe. quadrispinulatum, and Oe. dentatum (1). Other studies (2) have shown that the Oesophagostomum population of the caecum of the host is dominated numerically by Oe. quadrispinulatum. The present investigation was designed to supply quantitative data in support of these observations.

Examinations were made of the large intestines of ten sows, three Scottish and seven Danish, during the period February 1965 to February 1966. Nine were chosen at random from the available material. The tenth, a sow from which Oesophagostomum individuals were being expelled, was selected specially so that a comparison could be made between nematode populations in equilibrium and one undergoing spontaneous displacement from the normal habitat. In each case the caecum was separated from the colon and the latter divided into ten to twelve sections each measuring about half a meter in length. The nematodes in the lumen of each section were recovered, preserved, identified and counted. The worms were measured by projecting their image on a screen and using an opisometer to establish their lengths.

Oe. quadrispinulatum clearly favours a site closer to the ileocaecal valve than does Oe. dentatum (Fig. 1). The territories occupied by each species were observed, however, to overlap to varying degrees, Oe. quadrispinulatum sometimes extending into a more distal predilection site, and Oe. dentatum sometimes taking a more proximal position. Attempts were made to correlate these variations with 1) the absolute magnitude of the Oesophagostomum populations; 2) the relative numbers of each Oesophagostomum species; 3 ) the presence or absence of Trichuris suis; 4) the reproductive status of the sow. No constant pattern could be determined. 

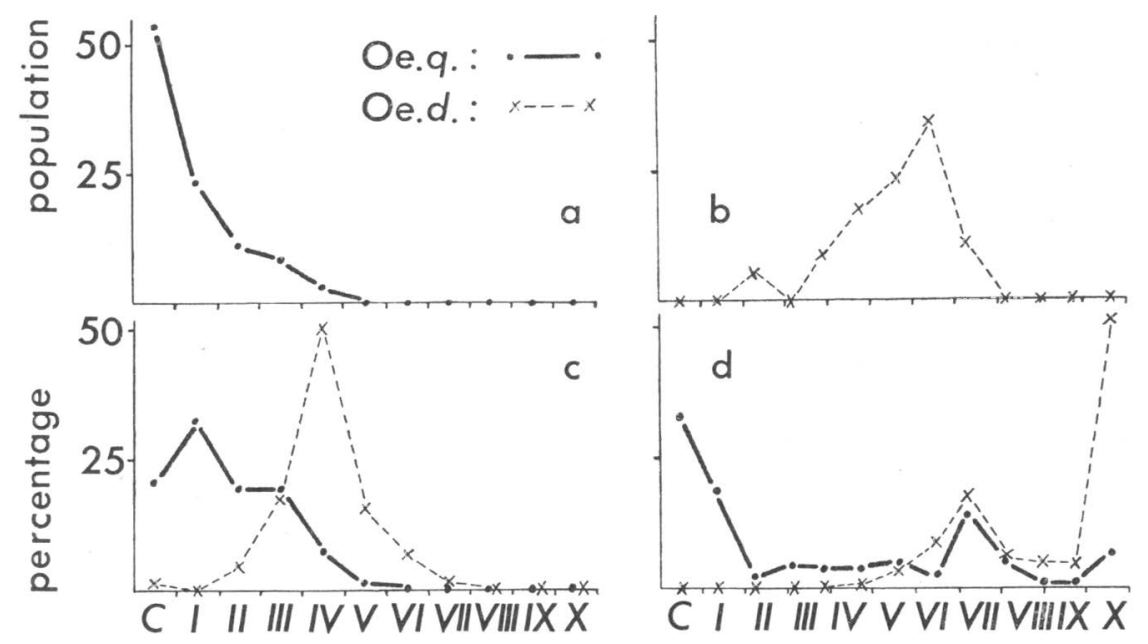

Figure 1. Linear distribution of Oesophagostomum spp. in the large intestine of the pig. $a$ and $b$ : monospecific infections. $c$ : mixed infection. $\mathrm{d}$ : spontaneous population expulsion. $(\mathrm{C}=$ caecum; I, II, III etc. $=$ sections of colon and rectum. Oe. q. $=$ Oe. quadrispinulatum. Oe. d. = Oe. dentatum).

The results in Fig. 1 are expressed in terms of the percentage of each Oesophagostomum population in each segment of the intestinal canal. Similar curves are produced by plotting the density of each Oesophagostomum species per unit area of mucosal surface against the distance from the ileocaecal valve, so that the distribution of Oesophagostomum species along the large intestine appears to be independent of the surface area or volume of luminal contents available in each section.

Predictably, the males and females of each species occupied similar positions in the intestine. Pairs of Oesophagostomum were often found in coitu, and in each case mating involved males and females of the same species.

Measurement of over 2.000 Oesophagostomum spp. individuals did not reveal any significant difference in the lengths of the worms taken from each section of the large intestine, nor did microscopical examination show any morphological differences which might indicate a progressively older population in the more distal segments (as is seen, for example, in Trichonema infections in horses (3)). 
When spontaneous expulsion of a population occurs, it naturally disrupts the customary distribution beyond recognition (Fig. 1).

D. E. Jacobs

(Holder of the Carlsberg-Wellcome Travelling Fellowship 1965/66), The Zoological Institute, University of Copenhagen, Denmark.

Present address: Department of Veterinary Pathology, University of Glasgow, Scotland, U. K.

\section{REFERENCES}

1. Dunn, A. M. \& D. E. Jacobs: Records of pig helminths in Great Britain and Denmark. Vet. Rec. 1966, 79, 156.

2. Nickel, E.-A. \& W. Haupt: Verlauf und Auswirkungen experimenteller Knötchenwurminvasionen bei Schweinen. Berl.Münch. tierärztl. Wschr. 1964, 77, 193-197.

3. Mathieson, A. O.: A study into the distribution of, and tissue responses associated with, some internal parasites of the horse. Thesis. University of Edinburgh, 1964.

(Received July 17, 1967). 